\title{
Impact of updated HER2 testing guidelines in breast cancer-re-evaluation of HERA trial fluorescence in situ hybridization data
}

\author{
Oliver C Stoss ${ }^{1,8}$, Andreas Scheel ${ }^{2,8}$, Iris Nagelmeier ${ }^{3}$, Hans-Ulrich Schildhaus ${ }^{4}$, \\ Thomas Henkel ${ }^{1}$, Giuseppe Viale ${ }^{5}$, Bharat Jasani ${ }^{6}$, Michael Untch ${ }^{7}$ and Josef Rüschoff ${ }^{1,3}$ \\ ${ }^{1}$ Targos Molecular Pathology GmbH, Kassel, Germany; ${ }^{2}$ Institute of Pathology, University of Cologne, Cologne, \\ Germany; ${ }^{3}$ Institute of Pathology Nordhessen, Kassel, Germany; ${ }^{4}$ Institute of Pathology, University of \\ Göttingen, Göttingen, Germany; ${ }^{5}$ Department of Pathology, University of Milan and European Institute of \\ Oncology, Milan, Italy; ${ }^{6}$ Nazarbayev University School of Medicine, Astana, Kazakhstan and ${ }^{7}$ Clinic for \\ Gynecology and Obstetrics, Helios Klinikum Berlin-Buch, Berlin, Germany
}

\begin{abstract}
Recently the American Society of Clinical Oncology and the College of American Pathologists have updated their clinical practice guidelines for HER2 testing in breast cancer. In order to evaluate these new recommendations, we have re-assessed the HER2 status of 6018 breast cancer cases of the screening population for the HERceptin adjuvant (HERA) trial that were originally centrally tested by fluorescence in situ hybridization based on the FDA-released test guidelines. According to the most recent 2013 ASCO/CAP recommendations, $3380(56.2 \%)$ cases were classified as HER2 positive compared with $3359(55.8 \%)$ applying the HERA/FDA scheme and 3339 (55.5\%) applying the 2007 ASCO/CAP guidelines. Twenty-one cases switched from negative (HERA/FDA scheme) to positive (2013 ASCO/CAP guidelines). This group is characterized by a mean HER2 gene copy number of $\geq 6.0$, polysomy or co-amplification of CEP17 with an average CEP17 count of 5, and with HER2 receptor overexpression in $75 \%$ of cases. On the basis of the HER2 gene copy number alone, we observe 494 cases $(8.2 \%)$ that are in the equivocal range. Most of these cases $(>80 \%)$ were also nondecisive by immunohistochemistry (score 2+) irrespective of whether ratio was $<\mathbf{2 . 0}>$. The number of equivocal cases that would require HER2 reflex testing decreases to $113(1.9 \%)$ if in addition to the HER2 gene copy number also the ratio of HER2 and CEP17 copy numbers is considered via dual-color in situ hybridization. The combination of applying the HER2 mean gene copy number as well as the HER2/CEP17 ratio to define equivocal test decisions by fluorescence in situ hybridization as proposed by the current ASCO/CAP guidelines appears to be a more optimum approach to adopt in order to avoid or minimize reporting of false negative results. Using the mean HER2 gene copy number alone for decision making results in a significant increase of equivocal cases.
\end{abstract}

Modern Pathology (2015) 28, 1528-1534; doi:10.1038/modpathol.2015.112; published online 25 September 2015

Accurate testing of HER2/neu protein level by immunohistochemistry and/or HER2 gene copynumber status by in situ hybridization is a prerequisite for effective anti-HER2 therapy in breast and gastric cancer. ${ }^{1,2}$ Thereby, determination of HER2 status is an integral part of the diagnostic work-up for sub-typing breast cancer and for clinical therapy decision making. ${ }^{3}$

Correspondence: Dr OC Stoss, PhD, Service Operations, Targos Molecular Pathology GmbH, Germaniastr. 7, 34119 Kassel, Germany.

E-mail: Oliver.Stoss@targos-gmbh.de

${ }^{8}$ These authors contributed equally to this work.

Received 17 June 2015; accepted 3 August 2015; published online 25 September 2015
Most of the previous HER2-directed therapy studies were based on HER2 positivity criteria described in FDA-approved test kits employed ('FDA criteria'). The cutoff value of 2.0 was established during certification of the PathVysion dual-color fluorescence in situ hybridization kit in 2002. ${ }^{4}$ Accordingly, in most of the approved dualcolor in situ hybridization tests patients are eligible for HER2-directed treatment if the HER2/CEP17 ratio is $\geq 2.0$ (http://www.fda.gov/MedicalDevices/ ProductsandMedicalProcedures/InVitroDiagnostics/ ucm301431.htm). The positivity cutoff for the mean HER2 gene copy number was initially set at $>4.0$ by the Inform Oncor monocolor fluorescence in situ hybridization test which was approved in 1997 for prognostic purposes (http://www.accessdata.fda. 
gov/scripts/cdrh/cfdocs/cfpma/pma.cfm?id = 95). Later on, FDA approved a monocolor chromogenic assay (SpoT-Light, Invitrogen, USA) for predictive purposes and herein the cutoff for the mean HER2 gene copy number was set at $>5$ (http://www.fda. gov/NewsEvents/Newsroom/PressAnnouncements/ 2008/ucm116918.htm). If HER2 immunohistochemistry tests were used, positivity was defined by intense ring-shaped immunostaining in $>10 \%$ of tumor cells (HercepTest, Dako, Denmark) (reviewed in Hanna et $a l^{5}$ ). In 2007, ASCO/CAP recommendations were published which defined a HER2/CEP17 ratio of $>2.2$ as HER2 positive and also introduced an equivocal range of $1.8-2.2 .^{6}$

Recently, ASCO/CAP have recommended new cutoff thresholds for definition of positivity for both immunohistochemistry and in situ hybridization, ${ }^{7}$ based on the refinement of the former 2007 criteria. Most importantly separate cutoffs for mono- and dual-color in situ hybridization are given. Tumors with a HER2/CEP17 ratio $\geq 2.0$ and those with a mean HER2 gene copy number $\geq 6.0$ are considered eligible for HER2-directed therapy. Thereby cases with a ratio $\geq 2.0$ are considered HER2 positive irrespective of the mean HER2 gene copy number putting this criterion as the most decisive. In addition, much more emphasis is put on quality assurance issues such as the consideration of histopathological breast cancer subtypes and tumor grading.

In this study, we revisited the primary HER2 testing data of the HERceptin adjuvant (HERA) screening population ${ }^{1,8}$ with regard to FDA and ASCO/CAP scoring criteria and to the concordance of fluorescence in situ hybridization HER2/CEP17 ratio and the mean HER2 gene copy number.

\section{Materials and methods}

\section{Patients and Tumor Samples}

The presented data are derived from the screening population of the HERA study which was an international, multicenter, randomized trial that compared 1 or 2 years of trastuzumab with observation alone in patients with HER2-positive breast cancer in the adjuvant setting. ${ }^{1,8}$ Tissue blocks or tissue slide sets from 10859 locally pre-tested patients with positive or inconclusive HER2 results were sent to the central laboratory Targos Molecular Pathology GmbH, Kassel, Germany for either HER2 immunohistochemistry and/or fluorescence in situ hybridization analysis, depending on the request of the respective local site. On the basis of this test algorithm, a total of 5174 patient samples have been analyzed prospectively by HER2 fluorescence in situ hybridization. In addition to the prospectively obtained data, 844 additional fluorescence in situ hybridization analyses were conducted retrospectively at Targos and European Institute of Oncology (Milan, Italy) for HERA samples where an immunohistochemistry result existed without centrally confirmed fluorescence in situ hybridization. ${ }^{8}$ Thus, a total of 6018 HERA patient samples were analyzed by HER2 fluorescence in situ hybridization. Of these, 3089 samples were also analyzed by immunohistochemistry.

\section{Analysis of HER2 Protein and Gene Status}

Immunoistochemistry (HercepTest Dako, Glostrup, Denmark) was performed according to the manufacturer's instructions, with the exception that an oil bath was used to heat the pretreatment buffer instead of a water bath. Fluorescence in situ hybridization staining was performed using the PathVysion HER2 DNA Probe Kit (PathVysion, Abbott Molecular, Des Plaines, Illinois, USA) according to the manufacturer's instructions.

\section{HER2 Evaluation}

HER2 immunohistochemistry scoring was performed according to the HercepTest guidelines (package insert, DAKO, Glostrup, Denmark) classifying a tumor as HER2 negative (Immunohistochemistry scores 0,1+), equivocal (score 2+) or positive (score $3+$ ). Immunohistochemistry positivity was defined by strong complete membrane staining in $>10 \%$ of tumor cells. Fluorescence in situ hybridization scoring was performed according to the PathVysion package insert guidelines determining the mean value of HER2 and CEP17 signals in 60 contiguous invasive tumor cells. Mean values of the HER2 gene copy number and the HER2/CEP17 ratio were calculated and patients with HER2/CEP17 ratios $\geq 2.0$ were considered eligible for treatment with trastuzumab.

For the comparison with the cutoff definitions by ASCO/CAP guideline recommendations, ${ }^{6,7}$ raw data of HER2 gene count and CEP17 counts per cell were used. An overview about the different thresholds is given in Table 1. The term 'polysomy' in this manuscript refers to the definition of $\geq 3$ CEP17 signal counts per cell calculated by the arithmetic mean per case. ${ }^{9}$ Homogenous protein expression was defined as $\geq 80 \%$ of tumor cells that express HER2. Focal protein expression was defined as $\leq 30 \%$ of tumor cells that express HER2. Consequently, HER2 expression in 31-79\% of tumor cells was defined as heterogeneous protein expression.

\section{Statistical Evaluation}

Data analysis was performed using the Targos HERA database (closure 14 February 2006) by 'R' statistical programming language version 2.13.1 (available under the GNU General Public License at http:// www.R-project.org). Mean values with s.d. were calculated for HER2 and CEP17 counts as well as HER2/CEP17 ratio. 
Table 1 Overview about HER2 fluorescence in situ hybridization thresholds for dual-color assays assessing HER2 and CEP17 gene copy number

\begin{tabular}{llll}
\hline & PathVysion package insert & ASCO/CAP 2007 (ref. 9) & ASCO/CAP 2013 (ref. 10) \\
\hline HER2 gene amplification & HER2/CEP17 ratio $\geq 2.0$ & HER2/CEP17 ratio $>2.2$ & $\begin{array}{l}\text { HER2/CEP17 } \geq 2.0 \text { or HER2/CEP17 }<2.0 \text { and } \\
\text { mean HER2 gene copy number } \geq 6.0\end{array}$ \\
& Not applicable & HER2/CEP17 ratio of & $\begin{array}{l}\text { HER2/CEP17 }<2.0 \text { and mean HER2 gene copy } \\
\text { number } \geq 4.0 \text { and }<6.0\end{array}$ \\
$\begin{array}{l}\text { No HER } \\
\text { amplification }\end{array}$ & HER2/CEP17 $<2.0$ & HER2/CEP17 ratio $<1.8$ & $\begin{array}{l}\text { HER2/CEP17 }<2.0 \text { and mean HER2 gene copy } \\
\text { number }<4.0\end{array}$ \\
\hline
\end{tabular}

\section{Results}

A total of 6018 HERA screening cases have been initially evaluated by dual-color fluorescence in situ hybridization using the FDA-approved package insert of the PathVysion test kit. We applied the modified thresholds for HER2 dual-color in situ hybridization recommended in the ASCO/CAP guidelines published in 2007 (ref. 6) and 2013 (ref. 7) on the same raw data. The different thresholds are summarized in Table 1.

\section{HER2/CEP17 Ratio: ASCO/CAP 2013 Criteria Applied to 6018 HERA Screening Cases}

The HER2 gene amplified cases according to the updated ASCO/CAP 2013 guidelines can be divided into four distinct subgroups (Table 2). The first subgroup defined with a ratio of $\geq 2.0$ and a gene count of $\geq 6(48.7 \%, n=2931)$ includes 353 cases $(12 \%)$ showing $\geq 3$ CEP17 signal counts per cell and an average CEP17 count of 2.2. From 1546 cases of this subgroup, HER2 immunohistochemistry was assessed and two third of the cases were scored positive. From 720 of the 1546 cases, the percentage of stained cells was determined. A total of 445 $(83.4 \%)$ of all 533 immunohistochemistry 3+ cases showed a homogenous HER2 expression over the tumor area. Twenty $(3.8 \%)$ of the immunohistochemistry positive cases showed focal staining and $25(13.4 \%)$ of all 187 immunohistochemistry $2+$ cases were focally expressed.

The intermediate level amplification subgroup (ratio of $\geq 2.0$ and a gene count of $\geq 4$ and $<6$ ) consisted of 381 cases $(6.3 \%)$. In comparison to the high-level amplification subgroup, no polysomic cases were identified and the average CEP17 count declined to 1.8. From 195 cases assessed by immunohistochemistry, the positive cases dropped to $13.3 \%$ $(n=26)$ within this subgroup whereas the amount of equivocal cases increased to $85.7 \%(n=167)$. Assessment of the percentage of stained cells from 79 cases showed a decrease of homogenously stained tumor cells to $63.8 \%$ (7 out of 11 cases) that were scored $3+$ whereas focal expression with a $3+$ score increased to $18.1 \%$ ( 2 out of 11 cases). With 13 out of 68 cases
(19.1\%), focal expression with a 2+ score remained on a relatively high level.

The low-level amplification subgroup (ratio of $\geq 2.0$ and a gene count of $<4$ ) included 47 cases $(0.8 \%)$. Thirty-nine $(83 \%)$ of these cases had a HER2/ CEP17 ratio of $<3$. No polysomy was observed, the average CEP17 count declined to 1.5. Thirty cases were characterized by immunohistochemistry. An inversion of the relationship of positive and equivocal immunohistochemistry cases was observed in comparison to the high-level amplification subgroup, with $10(33.3 \%)$ positive and 20 $(66.7 \%)$ equivocal cases. An interesting characteristic of the low-level amplification subgroup is that the immunohistochemistry $3+$ cases in this subgroup showed only homogenous protein expression of the tumor.

The last subgroup of the fluorescence in situ hybridization positive cases has been scored negative by the FDA guidelines since the ratio is $<2.0$. However, there were 21 cases with a gene count of $\geq 6$ which are now defined as positive according to the ASCO/CAP guidelines of 2013. A common feature of this subgroup is that all cases also show an increased CEP17 count (average count of 5.0). Twenty cases were assessed by immunohistochemistry and $15(75 \%)$ of these cases were scored as HER2 immunohistochemistry positive, supporting the ASCO/CAP recommendation. From nine of these positive cases, the percentage of stained tumor cells was assessed. Six cases (66.7\%) were homogenously expressed and three patient samples (11.1\%) showed focal HER2 expression.

From the 2525 patient samples $(41.9 \%)$ that were tested to be HER2 negative by fluorescence in situ hybridization according to the ASCO/CAP guidelines of 2013, we observed $135(5.3 \%)$ cases with an average CEP17 count of $\geq 3.0$. Most cases were disomic (CEP17: $2.2 \pm 0.5$ ). Immunohistochemistry was positive in $2 \%$ (24 of 1211 available immunohistochemistry stains) but none of these cases had a HER2 copy number $>4$. Fourteen of these cases had CEP17 counts $\geq 3.0$. Most of the equivocal immunohistochemistry cases assessed in this subgroup showed a heterogenous protein expression $(n=6$; $50 \%)$ followed by a focal staining of tumor cells $(n=4 ; 33.3 \%)$. 
Table 3 Comparison of HER2 fluorescence in situ hybridization results according to the original HERA classification (FDAapproved guidelines), the ASCO/CAP guidelines of 2007 and the ASCO/CAP guidelines of 2013

\begin{tabular}{lccc}
\hline & & ASCO/CAP & ASCO/CAP \\
& FDA & 2007 & 2013 \\
\hline $\begin{array}{l}\text { FISH positive } \\
\text { cases }\end{array}$ & $3359(55.8 \%)$ & $3339(55.5 \%)$ & $3380(56.2 \%)$ \\
$\begin{array}{l}\text { FISH equivocal } \\
\text { cases }\end{array}$ & NA & $44(0.7 \%)$ & $113(1.9 \%)$ \\
$\begin{array}{l}\text { FISH negative } \\
\text { cases }\end{array}$ & $2659(44.2 \%)$ & $2635(43,8)$ & $2525(41.9 \%)$ \\
\end{tabular}

Abbreviation: FISH, fluorescence in situ hybridization.

The numbers represent the amount of patients that have been tested positive, equivocal or negative.

\section{Comparison of HERA Screening Fluorescence In Situ Hybridization Results Obtained with the FDA, ASCO/ CAP 2007 and ASCO/CAP 2013 Guidelines}

Next, we compared the final fluorescence in situ hybridization results between the original HERA classification (FDA-approved kit guidelines), the ASCO/CAP guidelines of 2007 (ref. 6) and the most recent ASCO/CAP guidelines of $2013 .{ }^{7}$ In this comparison, the ASCO/CAP guidelines of 2013 produce the highest amount of fluorescence in situ hybridization positive cases $(n=3380$ cases; $56.2 \%)$ compared with 3359 cases (55.8\%) using the FDA guideline (Table 3 ). The above mentioned 21 cases called positive according to the ASCO/CAP 2013 recommendations (average HER2 copy number $\geq 6$ ) would have been scored negative according to the original HERA testing guidelines. However, since the HERA trial screening algorithm also allowed the site for requesting central immunohistochemistry testing as the first choice, the number of patients included into the HERA trial would have differed by only five cases if the new ASCO/CAP criteria would have been applied and if the sequential algorithm (immunohistochemistry first followed by in situ hybridization) would have been followed for equivocal cases which is widely implemented in routine pathology.

\section{Investigation of Equivocal Cases}

The most obvious difference between the FDA and the ASCO/CAP guidelines is the definition of the equivocal range. The FDA fluorescence in situ hybridization scoring criteria do not foresee a final equivocal result although the recommendation is given to re-assess cases with a ratio between 1.8 and 2.2 on 40 nuclei. The former ASCO/CAP guidelines published in 2007 defined cases with a ratio between 1.8 and 2.2 as equivocal. In total 44 cases $(0.7 \%)$ of all 6018 HERA cases assessed by fluorescence in situ hybridization would have been determined equivocal (Table 3). 
Table 4 Comparison of the mean HER2 gene copy number and the HER2/CEP17 ratio on the HERA screening population

\begin{tabular}{|c|c|c|c|c|}
\hline & Gene count positive $(\geq 6)$ & Gene count equivocal (4-6) & Gene count negative $(<4)$ & Total \\
\hline Ratio positive (>2.2) & 2922 & 374 & 43 & 3339 \\
\hline Ratio equivocal $(1.8-2.2)$ & 13 & 17 & 14 & 44 \\
\hline Ratio negative $(<1.8)$ & 17 & 103 & 2515 & 2635 \\
\hline Total & 2952 & 494 & 2572 & 6018 \\
\hline
\end{tabular}

Numbers represent amount of patients.

The definition of equivocal cases according to the recent ASCO/CAP guidelines of 2013 has changed by focusing on the copy number instead of the ratio. The amount of equivocal fluorescence in situ hybridization cases increased to 113 cases $(1.9 \%)$. Sixty-nine $(61.1 \%)$ of all cases had $\geq 3$ CEP17 signal counts per cell with an average CEP17 count of 3.2. The equivocal group was analyzed by immunohistochemistry on 84 cases. Sixty-nine patient samples $(82.1 \%)$ were again scored as equivocal $(2+)$ and remained nondecisive. Twelve cases (14.3\%) were HER2 positive based on immunohistochemistry.

For single-probe in situ hybridization assays where the decision is based on the gene count only, the amount of equivocal cases (gene count $\geq 4$ and $<6$ ) would have increased dramatically to 494 cases $(8.2 \%$, Table 4). Interestingly, most of these cases $(>80 \%)$ were nondecisive by immunohistochemistry (score 2+) irrespective of whether the ratio was $<2.0>$. HER2 status could be clarified in about $15 \%$ of these cases, only.

Another observation is that only $0.3 \%$ of all cases $(n=17)$ fall into the equivocal subgroup both by HER2 gene counts and HER2 ratio (Table 4). A total of $5.9 \%$ of these cases had CEP17 counts $\geq 3.0$, comparable to the negative group. One of eight available immunohistochemistry stains for this group was immunohistochemistry positive; this case had a HER2 gene count of 5.97 .

\section{Discussion}

In the past few years, a continuous improvement of the accuracy of HER2 testing has been observed. ${ }^{10-13}$ As a part of this process, the American Society of Clinical Oncology and the College of American Pathologists have recently updated the recommendations for HER2 testing in breast cancer. Besides improved technical guidelines for standardization and test performance, the expert panel modified the interpretation guidelines for both in situ hybridization and immunohistochemistry. Although the former ASCO/CAP guidelines focused more on the exclusion of false positive cases, the application of the current guidelines favors the exclusion of false negative HER2 results.

In this study, we applied the most recent ASCO/ CAP guidelines for fluorescence in situ hybridization on one of the largest HER2 fluorescence in situ hybridization series in 6018 breast cancer specimens of the screening population of the HERA trial. ${ }^{1}$ The number of patients included into the HERA trial would have differed by only five cases if the new ASCO/CAP 2013 criteria would have been applied. These cases are scored fluorescence in situ hybridization positive according to the new guidelines because the mean HER2 gene copy number was higher than 6 . These five cases have been excluded from the HERA trial because the immunohistochemistry was equivocal and the fluorescence in situ hybridization HER2/CEP17 ratio was $<2$. The HERA fluorescence in situ hybridization data support the ASCO/CAP recommendation of receiving a HER2 targeted therapy in case the mean HER2 gene copy number is equal or higher than 6 irrespective of the ratio with up to $75 \%$ of all cases being also immunohistochemistry positive. Dowsett et al. ${ }^{8}$ could show that the degree of HER2 amplification in HERA was not associated with therapy response. Patients with a low HER2 amplified tumor (mean HER2 gene copy number $4 \leq$ and $\leq 9$ ) had identical outcome compared with patients with highly amplified tumors (mean HER2 gene copy number $>9$ ). This observation implies that patients with a mean HER2 gene copy number between 4 and 6 might have a chance to profit from HER2-directed therapy irrespective of the ratio which is now reflected in the current ASCO/CAP guidelines.

Re-evaluation of the HERA trial data disclosed a high agreement between fluorescence in situ hybridization results obtained by HER2/CEP17 ratio and mean HER2 gene copy number (Table 4). The discrepancies were mainly caused by the definition of the equivocal range. The FDA criteria did not foresee any final equivocal result. The former ASCO/ CAP 2007 guidelines defined the equivocal range based on the HER2/CEP17 ratio which results in 44 equivocal cases. The new ASCO/CAP guidelines define the equivocal range for dual-color in situ hybridization on a combination of HER2/CEP17 ratio and the mean HER2 gene copy number resulting in 113 equivocal cases (1.9\%). About 14\% are determined as HER2 positive using immunohistochemistry reflex testing whereas $>80 \%$ of cases remain equivocal. The amount of cases to be re-investigated a second time would increase dramatically to 494 equivocal cases $(8.2 \%)$ if the HER2 mean copy number would have been used alone as this is the 
case for single gene monocolor in situ hybridization, requiring an $11 \mathrm{x}$ increase in additional immunohistochemistry testing and hindering clinical decision making. The ASCO/CAP decision to combine both the HER2 ratio and the mean HER2 gene copy number to define the equivocal range for dual-color in situ hybridization seems to be a valid compromise between reducing the false negative rate and not unnecessarily enforcing a retest.

We analyzed the distribution of CEP17 signals in more detail. The percentage of tumors with a mean CEP17 count of $\geq 3.0$ (so-called polysomy rate) and the mean absolute CEP17 per case count were assessed. All tumors negative or equivocal by HER2 ratio but positive based on the mean HER2 gene copy number showed CEP17 counts $\geq 3.0$. Recent studies using CGH indicate that true polysomy, i.e., a gain of extra copies of the whole chromosome 17, is a rare event $^{14}$ (review in Hanna et $a 5^{5}$ ). Complex rearrangements of chromosome 17 were often observed and the locus bound by the CEP17 probe may be amplified together with the HER2 locus or independently. In our data, increased CEP17 counts are coupled with high HER2 gene count (Table 2). Clinical evidence emerged that the CEP17 count on its own has no predictive value for HER2-directed therapy ${ }^{8,15}$ whereas the value of the CEP17 count for response prediction of anthracyclines is still under debate. ${ }^{16}$

Our data (Table 2) show a high immunohistochemistry positivity rate among the cases with a high mean HER2 gene copy number and a low ratio. The average percentage of HER2 immunohistochemistry $3+$ cases is higher than $66 \%$ for cases showing a mean HER2 gene copy number $\geq 6$, irrespective of the ratio. This observation is supported by the fact that it was recently shown by image analysis that immunohistochemistry is more closely related to the mean HER2 copy number than to ratio values in 3401 investigated HERA trial cases. ${ }^{17}$

In summary, the retrospective application of the new ASCO/CAP 2013 guidelines on the HERA fluorescence in situ hybridization data results in only few changes of included patients compared with the FDA guideline. The in situ hybridization evaluation methods calculating the HER2/CEP17 ratio and absolute HER2 gene copy number show a high degree of concordance yet are not interchangeable. The determination of the mean HER2 gene copy number correlates more closely with the HER2 immunohistochemistry results whereas the ratio method is more powerful in discriminating positive from negative cases. Abnormal CEP17 numbers cause discrepancies between the two techniques. A high CEP17 count ( $\geq 3.0$ copies) may justify preferring the absolute count as the majority of those ratiolow but count-high cases are immunohistochemistry positive.

\section{Acknowledgments}

The HERA study was sponsored by F. Hoffmann-La Roche Ltd. The HERA trial was conducted in collaboration with the Breast International Group (BIG), the BrEAST Data Center and Frontier Science, Scotland.

\section{Disclosure/conflict of interest}

GV and JR received travel grants and honoraria (advisory boards) from Roche. The remaining authors declare no conflict of interest.

\section{References}

1 Piccart-Gebhart MJ, Procter M, Leyland-Jones B et al. Herceptin Adjuvant (HERA) Trial Study Team: trastuzumab after adjuvant chemotherapy in HER2-positive breast cancer. N Engl J Med 2005;353:1659-1672.

2 Bang YJ, Van Cutsem E, Feyereislova A et al. Trastuzumab in combination with chemotherapy versus chemotherapy alone for treatment of HER2-positive advanced gastric or gastro-oesophageal junction cancer (ToGA): a phase 3, open-label, randomised controlled trial. Lancet 2010;376:687-697.

3 Goldhirsch A, Wood WC, Coates AS et al. Strategies for subtypes-dealing with the diversity of breast cancer: highlights of the St. Gallen International Expert Consensus on the Primary Therapy of Early Breast Cancer 2011. Ann Oncol 2011;22:1736-1747.

4 United States Food and Drug Administrator, Device Approvals and Clearances, "PathVysion; HER-2 DNA Probe Kit - P980024/S001", Issued December 31, 2001, Updated April 25, 2002: www.accessdata.fda.gov/ cdrh_docs/pdf/P980024S001c.pdf.

5 Hanna WM, Rüschoff J, Bilous $\mathrm{M}$ et al. HER2 in situ hybridization in breast cancer: clinical implications of polysomy 17 and genetic heterogeneity. Mod Pathol 2014;27:4-18.

6 Wolff AC, Hammond ME, Schwartz JN et al. American Society of Clinical Oncology/College of American Pathologists guideline recommendations for human epidermal growth factor receptor 2 testing in breast cancer. J Clin Oncol 2007;25:118-145.

7 Wolff AC, Hammond ME, Hicks DG et al. Recommendations for human epidermal growth factor receptor 2 testing in breast cancer: American Socienty of Clinical Oncology/College of American Pathologists clinical practice guideline update. J Clin Oncol 2013;31:3997-4013.

8 Dowsett M, Procter M, McCaskill-Stevens W et al. Disease-free survival according to degree of HER2 amplification for patients treated with adjuvant chemotherapy with or without 1 year of trastuzumab: the HERA Trial. J Clin Oncol 2009;27:2962-2969.

9 Marchiò C, Lambros MB, Gugliotta $\mathrm{P}$ et al. Does chromosome 17 centromere copy number predict polysomy in breast cancer? A fluorescence in situ hybridization and microarray-based CGH analysis. J Pathol 2009;219:16-24.

10 Rakha EA, Starczynski J, Lee AH et al. The updated ASCO/CAP guideline recommendations for HER2 testing in the management of invasive breast cancer: a critical review of their implilcations for routine practice. Histopathology 2014;64:609-615. 
11 Perez EA, Suman VJ, Davidson NE et al. HER2 testing by local, central and reference laboratories in specimens from the North Central Cancer Treatment Group N9831 intergroup adjuvant trial. J Clin Oncol 2006;24: 3032-3038.

12 Middleton LP, Price KM, Puig P et al. Implementation of American Society of Clinical Oncology/College of American Pathologists HER2 Guidelines Recommendations in a tertiary care facility increases HER2 immunohistochemistry and fluorescence in situ hybridization concordance and decreases the number of inconclusive cases. Arch Pathol Lab Med 2009;133:775-780.

13 Chen X, Yuan Y, Gu Z et al. Accuracy of estrogen receptor, progesterone receptor and HER2 status between core needle and open excision biopsy in breast cancer: a meta-analysis. Breast Cancer Res Treat 2012;114:957-967.
14 Gunn S, Yeh IT, Lytvak I et al. Clinical array-based karyotyping of breast cancer with equivocal HER2 status resolves gene copy number and reveals chromosome 17 complexity. BMC Cancer 2010;10:396.

15 Vanden Bempt I, Van Loo P, Drijkoningen M et al. Polysomy 17 in breast cancer: clinicopathologic significance and impact on HER-2 testing. J Clin Oncol 2008;26:4869-4874.

16 Slamon DJ, Press MF. Alterations in the TOP2A and HER2 genes: association with adjuvant anthracycline sensitivity in human breast cancers. J Natl Cancer Inst 2009;101:615-618.

17 Zabaglo L, Stoss O, Rueschoff J et al. HER2 staining intensity in HER2-positive disease: relationship with fluorescence in situ hybridization amplification and clinical outcome in the HERA trial of adjuvant trastuzumab. Ann Oncol 2013;24:2761-2766. 